\title{
AN AMBROSETTI-PRODI-TYPE PROBLEM FOR AN ELLIPTIC SYSTEM OF EQUATIONS VIA MONOTONE ITERATION METHOD AND LERAY-SCHAUDER DEGREE THEORY
}

\author{
D. C. DE MORAIS FILHO
}

\begin{abstract}
In this paper we employ the Monotone Iteration Method and the Leray-Schauder Degree Theory to study an $\mathbb{R}^{2}$-parametrized system of elliptic equations. We obtain a curve dividing the plane into two regions. Depending on which region the parameter is, the system will or will not have solutions. This is an Ambrosetti-Prodi-type problem for a system of equations.
\end{abstract}

\section{INTRODUCTION}

Let us consider a bounded domain $\Omega \subseteq \mathbb{R}^{N}, \quad N \geq 2$, with boundary $\partial \Omega$ of class $C^{2, \alpha}, 0<\alpha \leq 1$.

In what follows we shall denote the Cartesian product of Hölder spaces as $\left(C^{i, \alpha}\right)^{2}=C^{i, \alpha}(\bar{\Omega}) \times C^{i, \alpha}(\bar{\Omega}), 0<\alpha \leq 1, i=0,1,2$ and $\left(C^{\alpha}\right)^{2}=\left(C^{0, \alpha}(\bar{\Omega})\right)^{2}$.

Remark 0.1. $\left(C^{i, \alpha}\right)^{2} \hookrightarrow\left(C^{j, \alpha}\right)^{2}$ compactly if $i \geq j \geq 0$.

Let us consider the following system of partial differential elliptic equations

$$
\left\{\begin{aligned}
-\Delta u & =f(x, u, v)+h(x), \quad \Omega, \\
-\Delta v & =g(x, u, v)+l(x), \quad \Omega, \\
u & =v=0, \quad \partial \Omega,
\end{aligned}\right.
$$

where $h, l \in C^{\alpha}(\bar{\Omega})$ and $f, g \in C^{\alpha}(\bar{\Omega} \times \mathbb{R} \times \mathbb{R})$ are real functions. By a solution of $(S)$ we mean a vector-function $U=(u, v) \in\left(C^{2, \alpha}\right)^{2}$ satisfying both equations in $(S)$.

Any function $h \in C^{\alpha}(\bar{\Omega})$ can be uniquely decomposed as $h=t \phi_{1}+h_{1}$, where $\phi_{1}>0$ is an eigenfunction associated to the first eigenvalue $\lambda_{1}>0$

1991 Mathematics Subject Classification. Primary: 35J55.

Key words and phrases. Ambrosetti-Prodi problem, Monotone iteration method, LeraySchauder degree theory.

Partially sponsored by CNPq-Brazil.

Received: March 7, 1996. 
of the eigenvalue problem $\left(-\Delta, H_{0}^{1}(\Omega)\right), h_{1} \in C^{\alpha}(\bar{\Omega}), \int h_{1} \phi_{1}=0, \int \phi_{1}^{2}=1$ and $t=\int h \phi_{1}$ ( unless otherwise mentioned all integrals are taken over the whole $\Omega$ ).

According the above observations, system $(S)$ may be $\mathbb{R}^{2}$-parametrized, and for the convenience of notation, be rewritten in the matrix form

$$
(S)_{T} \quad\left\{\begin{aligned}
-\Delta U & =F(x, U)+T \phi_{1}+H_{1}(x), \quad \Omega, \\
U & =0, \partial \Omega
\end{aligned}\right.
$$

where $U=(u, v), T=\left(\begin{array}{l}r \\ s\end{array}\right) \in \mathbb{R}^{2}$,

$$
F(x, U)=\left(\begin{array}{l}
f(x, u, v) \\
g(x, u, v)
\end{array}\right) \quad \text { and } H_{1} \equiv\left(\begin{array}{l}
h \\
l
\end{array}\right) .
$$

We make use of the following notation: $N=\operatorname{span}\left\{\phi_{1}\right\}, N^{\perp}=\{f \in$ $\left.C^{\alpha}(\bar{\Omega}) ; \int f \phi_{1}=0\right\}$ and $N_{\perp}^{2}=N^{\perp} \times N^{\perp}$.

For any fixed $H_{1} \in N_{\perp}^{2}$ our main goal is to find the set of points $T \in \mathbb{R}^{2}$ for those, system $(S)_{T}$ has or has not solutions. Grosso modo, for this matter, with convenient hypotheses on $F$ which shall be timely introduced, we find a curve $\Gamma=\Gamma\left(H_{1}\right)$ splitting the plane into two disjoint unbounded domains $E$ and $N\left(\mathbb{R}^{2}=E \cup \Gamma \cup N\right)$ such that

(i) $\left(S_{T}\right)$ does not have a solution if $T \in N$

(ii) $\left(S_{T}\right)$ has at least one solution if $T \in \Gamma$

(iii) $\left(S_{T}\right)$ has at least two solutions if $T \in E$.

The paper is divided into two parts. In the first one we use the Monotone Iteration Method to find the first solution and construct the curve. Although some ideas of the first part already appear in [7], where we solved a kind of this problem using variational techniques, we decided to include them here for completeness of the text. In the second part, we obtain a priori estimates for eventual solutions of $(S)_{T}$ using the Hardy-Sobolev inequality (see [2] or [6]) and apply the Leray-Schauder degree theory to obtain the second solution. Then we find a solution for $T$ on the curve.

In this paper we have the advantage of relaxing the very restrictive hypothesis on the non-linearity $F=\nabla H$ ( for some vector-function $H$ ) which is imposed by the variational methods we used in [7]. Also relating to [7], another sharper result we found in this paper was the existence of a solution for $T$ on the curve $\Gamma$.

In the scalar case, the pioneering paper involving this kind of problem but with a very different formulation is [1]. Also in the scalar case, good references for this kind of problems up to 80's may be found in [4] and [8]. 


\section{FIRST PART}

1. Existence of a Region $\mathcal{R}$ in the Plane Where $(S)_{T}$ Has No SOlution

Let us order $\mathbb{R}^{2}$ with the partial order $Z=\left(z_{1}, z_{2}\right) \geq 0$ if and only if $z_{1,} z_{2} \geq 0$.

We shall assume the following growth conditions on the non-linearity $F$ :

$$
\begin{aligned}
& F(x, Z) \geq \underline{A} Z-C \\
& F(x, Z) \geq \bar{A} Z-C
\end{aligned}
$$

for all $x \in \Omega$ and $Z \in \mathbb{R}^{2}$, where

$$
\bar{A}=\left(\begin{array}{ll}
\bar{a} & \bar{b} \\
\bar{c} & \bar{d}
\end{array}\right), \underline{A}=\left(\begin{array}{ll}
\bar{a} & \bar{b} \\
\bar{c} & \bar{d}
\end{array}\right) \in M_{2 \times 2}(\mathbb{R}), \quad C=\left(\begin{array}{l}
c \\
c
\end{array}\right)>0
$$

and these matrices satisfy

$$
\begin{array}{ll}
\left(\underline{A}-\lambda_{1} I\right)^{-1} Z \leq 0 & \text { for all } Z \geq 0, Z \in \mathbb{R}^{2}, \\
\left(\bar{A}-\lambda_{1} I\right)^{-1} Z \geq 0 & \text { for all } Z \geq 0, Z \in \mathbb{R}^{2} .
\end{array}
$$

( $I=$ the identity of $M_{2 \times 2}(\mathbb{R})$ ).

It is immediate that condition (1.4) is equivalent to conditions

$$
\begin{gathered}
\underline{b}, \underline{c} \geq 0 \\
\left(\lambda_{1}-\underline{a}\right)\left(\lambda_{1}-\underline{d}\right)-\underline{b c}>0 \\
\underline{a}, \underline{d}<\lambda_{1}
\end{gathered}
$$

or to conditions

$$
\begin{gathered}
\underline{b}, \underline{c}<0 \\
\left(\lambda_{1}-\underline{a}\right)\left(\lambda_{1}-\underline{d}\right)-\underline{b c}<0 \\
\underline{a}, \underline{d}>\lambda_{1 .}
\end{gathered}
$$

On its turn, condition (1.5) is equivalent to conditions

$$
\begin{gathered}
\left(\lambda_{1}-\bar{a}\right)\left(\lambda_{1}-\bar{d}\right)-\bar{b} \bar{c}>0 \\
\bar{a}, \bar{d}>\lambda_{1}
\end{gathered}
$$

$$
\bar{b}, \bar{c} \leq 0
$$

or to conditions 


$$
\begin{gathered}
\bar{b}, \bar{c}>0 \\
\left(\lambda_{1}-\bar{a}\right)\left(\lambda_{1}-\bar{d}\right)-\bar{b} \bar{c}<0 \\
\bar{a}, \bar{d}<\lambda_{1} .
\end{gathered}
$$

Remark 1.1. If (1.6) holds, conditions (1.7) and (1.8) are equivalent to

$$
\underline{\mu}_{1}, \underline{\mu}_{2}<\lambda_{1} \text {, where } \underline{\mu}_{1}, \underline{\mu}_{2} \text { are the eigenvalues of } \underline{A} \text {. }
$$

Likewise, if (1.12) holds, conditions (1.13) and (1.14) are equivalent to

$$
\bar{\mu}_{1}, \bar{\mu}_{2}>\lambda_{1} \text {,where } \bar{\mu}_{1}, \bar{\mu}_{2} \text { are the eigenvalues of } \bar{A} \text {. }
$$

With the above hypotheses we have

Lemma 1.1. (Existence of a region in the plane $(r, s)$ where $(S)_{T}$ has no solution) Let (1.1), (1.2), (1.4) and (1.5) be assumed. Then, for a given $H_{1} \in\left(C^{\alpha}\right)^{2}$, there exists an unbounded domain $\mathcal{R}$ in the plane $(r, s)$ (depending only upon $\bar{A}, \underline{A}$ and $\lambda_{1}$ ) such that

$$
\text { if } T \in \mathcal{R} \text {, system }(S)_{T} \text { has no solution. }
$$

Proof. Suppose $U=(u, v)$ is a solution of $(S)_{T}$. Multiplying both equations of this system by $\phi_{1}$, integrating by parts and using (1.1) and (1.2) we achieve the inequalities

$$
\left(\underline{A}-\lambda_{1} I\right)\left(\begin{array}{l}
\gamma \\
\delta
\end{array}\right) \leq\left(\begin{array}{c}
-\widetilde{r} \\
-\widetilde{s}
\end{array}\right)
$$

and

$$
\left(\bar{A}-\lambda_{1} I\right)\left(\begin{array}{l}
\gamma \\
\delta
\end{array}\right) \leq\left(\begin{array}{c}
-\widetilde{r} \\
-\widetilde{s}
\end{array}\right)
$$

where $\gamma=\int u \phi_{1}, \delta=\int v \phi_{1}, \widetilde{r}=r-c$ and $\widetilde{s}=s-c$.

Applying (1.4) and (1.5) to (1.21) and (1.22), respectively, and using a straightforward computation we see that

(i) If $\gamma \leq 0$ then

$$
\widetilde{s} \leq\left\{\left(\underline{d}-\lambda_{1}\right) / \underline{b}\right\} \widetilde{r} \text { when } \underline{b} \neq 0 ; \text { or } \widetilde{r} \leq 0 \text { when } \underline{b}=0
$$

(ii) If $\gamma \geq 0$ then

$$
\widetilde{s} \geq\left\{\left(\bar{d}-\lambda_{1}\right) / \bar{b}\right\} \widetilde{r} \text { when } \bar{b} \neq 0 ; \text { or } \widetilde{r} \leq 0 \text { when } \bar{b}=0 \text {. }
$$

Hence, independently of the sign of $\gamma$, the pair $(\widetilde{r}, \widetilde{s})$ is in a region composed of the intersection of two half-planes, each of them is bounded above by a straight-line of negative or infinity slope passing through the origin. The set $\mathcal{R} \subset \mathbb{R}^{2}$ is the complement of this region in the original variables $r$ and $s$. 


\section{Existence of Sub and Supersolution}

The existence of a solution for $T$ in a certain region of $\mathbb{R}^{2}$ is guaranteed by the Monotone Iteration Method. This method requires the notions of sub and supersolution. We now give some definitions and remarks in order to go further.

Definition 2.1. A vector-function $U \in\left(C^{2, \alpha}\right)^{2}$ is a "subsolution" for the system $(S)$ if

$$
\left\{\begin{aligned}
-\Delta U & \leq F(x, u)+H(x), \quad \Omega, \\
U & =0, \quad \partial \Omega .
\end{aligned}\right.
$$

A "supersolution" is defined likewise by reversing the inequality.

Definition 2.2. We say that a matrix-function $A(x)$ "satisfies a maximum principle" if for all $U$ satisfying

$$
\left\{\begin{aligned}
-\Delta U & \geq A(x) U, \quad \Omega, \\
U & =0, \quad \partial \Omega
\end{aligned}\right.
$$

we have $U \geq 0$.

Remark 2.1. If $A$ is a cooperative matrix (i.e., $A$ has nonnegative off diagonal terms) the condition

$$
(A(x) Z, Z)_{\mathbb{R}^{2}} \leq \underline{\mu}, \forall x \in \bar{\Omega}, Z \in \mathbb{R}^{2},
$$

where $\mu<\lambda_{1}$ and $(., .)_{\mathbb{R}^{2}}$ is the usual $\mathbb{R}^{2}$ inner product, is a sufficient condition for $A$ to satisfy a maximum principle (see [10]). If $A$ is a symmetric matrix, condition (2.1) is equivalent to condition (1.18).

Remark 2.2. If the matrix $A$ has entries in the space $C^{\alpha}(\bar{\Omega})$ and satisfies a maximum principle, then the linear system

$$
\left\{\begin{aligned}
-\Delta U & =A(x) U+F(x), \quad \Omega \\
U & =0, \quad \partial \Omega
\end{aligned}\right.
$$

with $F \in\left(C^{\alpha}\right)^{2}$ has a (unique) solution. Indeed, in this case the above system has the uniqueness of solution property and this fact yields readily an $\left(C^{2, \alpha}\right)^{2}$ a priori estimates for its solutions. Hence by Remark 0.1, a Fredholm Alternative is valid for this system.

Let us prove the existence of sub and supersolutions for $(S)_{T}$.

Lemma 2.1. (Existence of subsolutions) Suppose that (1.1), (1.6), (1.7) and (1.8) hold. Then for a given vector-function $H=H_{1}+T \phi_{1} \in\left(C^{\alpha}\right)^{2}$ there exists a subsolution $\underline{U} \in\left(C^{2, \alpha}\right)^{2}$ of the system $(S)_{T}$ such that if $\bar{U}$ is any supersolution of this system, we have that

$$
\underline{U}<\bar{U} \quad \text { in } \Omega \quad \text { and } \quad \frac{\partial \bar{U}}{\partial \nu}<\frac{\partial \underline{U}}{\partial \nu} \quad \text { on } \quad \partial \Omega
$$

(here $\frac{\partial \mathrm{U}}{\partial \nu}=\left(\frac{\partial u}{\partial \nu}, \frac{\partial v}{\partial \nu}\right)$ and $\frac{\partial}{\partial \nu}$ is the outward directional derivative). 
Proof. Choose $C$ such that one has strict inequality in (1.1). Assertions (1.6), (1.7) and (1.8) give sufficient and necessary conditions for $A$ to satisfy a Maximum Principle (see [5]). Therefore by Remark 2.2 the system

$$
\left\{\begin{array}{rcc}
-\Delta W & = & \underline{A} W-C+H, \quad \Omega \\
W & = & 0, \quad \partial \Omega
\end{array}\right.
$$

has a unique solution $\underline{U}$. By (1.1) it is immediate that $\underline{U}$ is a subsolution for $(S)_{T}$. From (1.6), (1.8) and the choice of $C$, using the Maximum Principle and the Hopf Maximum Principle of the scalar case for both equations in the above system we assure that (2.3) holds.

Lemma 2.2. (Existence of supersolutions) For any given vector-function $H_{1} \in\left(N_{\perp}\right)^{2}$, there exists a $T_{o} \in \mathbb{R}^{2}, T_{o}<0$ (depending upon $\Omega$ and $H_{1}$ ) such that system $(S)_{T}$ has a positive supersolution $\bar{U}$ for all $T \leq T_{o}$.

Proof. Let us fix $N>0$ and define

$m>\left\{\max \left\{\left|f(x, \eta, \xi)+h_{1}(x)\right|\right\},\left\{\max \left\{\mid g(x, \eta, \xi)+l_{1}(x)\right\}, x \in \bar{\Omega} ;|s|,|\xi| \leq N\right\}\right.$

Consider the domains below to be chosen timely

$$
\Omega_{1} \subseteq \overline{\Omega_{1}} \subseteq \Omega_{2} \subseteq \overline{\Omega_{2}} \subseteq \Omega
$$

and denote $\delta=\operatorname{vol}\left(\Omega \backslash \Omega_{1}\right)$. Let us take $\vartheta \in C^{\alpha}(\bar{\Omega})$, such that $\vartheta \equiv m$ in $\Omega \backslash \Omega_{2}, \vartheta \equiv 0$ in $\Omega_{1}$ and $0 \leq \vartheta \leq m$. Let $\bar{w}$ be the solution of the equation

$$
\left\{\begin{aligned}
-\Delta \bar{w} & =\vartheta, \Omega \\
\bar{w} & =0, \partial \Omega
\end{aligned}\right.
$$

Hence, $\bar{w} \in C^{2, \alpha}(\bar{\Omega})$ and $\bar{w}>0$. From $L^{p}$ estimates and the Sobolev embedding Theorem it is possible to choose $\Omega_{1}$ such that $|\bar{w}(x)|<N$ for $x \in \bar{\Omega}$.

Now choosing $T_{o}=\left(r_{o}, s_{o}\right)$ with $r_{o}$ and $s_{o}$ sufficiently negative as to have

$$
\vartheta \geq m+r_{o} \phi_{1}, m+s_{o} \phi_{1}
$$

it is readily seen from the definition of $m$ that $\bar{U}=(\bar{w}, \bar{w})>0$ is the wanted supersolution.

\section{The Monotone Iteration Method}

This method has the advantage of being constructive and yields easily some important facts (see Corollary 3.1 and Lemma 5.2).

Theorem 3.1. (Monotone iteration method) Let $\underline{U}$ and $\bar{U}$ be, respectively, ordered sub and supersolution of $(S)$ with $\underline{U} \leq \bar{U}$. Let $A(x)$ be a matrixfunction with entries in the space $C^{\alpha}$ such that:

$$
F\left(x, Z_{2}\right)-F\left(x, Z_{1}\right) \geq A(x)\left(Z_{2}-Z_{1}\right), \quad Z_{2} \geq Z_{1} ; Z_{2}, Z_{1} \in \mathbb{R}^{2}, \quad x \in \bar{\Omega}
$$

and $Z_{2}, Z_{1} \in\left[\min _{x \in \bar{\Omega}} \underline{U}, \max _{x \in \bar{\Omega}} \bar{U}\right] \quad\left(\min _{x \in \bar{\Omega}} \underline{U}:=\min _{\left(\min _{x \in \bar{\Omega}} \underline{\underline{U}}\right.} \underline{\underline{U}}\right)$. The same definition for $\left.\max _{x \in \bar{\Omega}} \bar{U}\right)$. Then, system $(S)$ has two solutions $U$ and $V$ in $\left(C^{2, \alpha}\right)^{2}$ (eventually it may occur that $U \equiv V$ ) such that $\underline{U} \leq U \leq V \leq \bar{U}$. Moreover, if $W$ is 
any other solution with $W \in[\underline{U}, \bar{U}]$, then $W \in[U, V]$. (Here $[\underline{U}, \bar{U}] \equiv\{U \in$ $\left.\left.\left(C^{2, \alpha}\right)^{2}: \underline{U} \leq U \leq \bar{U}\right\}\right)$

Proof. By Remark 2.2 the following operator is well defined

$$
\begin{aligned}
\mathcal{T} & : \quad[\underline{U}, \bar{U}] \longrightarrow\left(C^{2, \alpha}\right)^{2} \\
U & \mapsto W=\mathcal{T} U,
\end{aligned}
$$

where $W$ is the (unique) solution of the system

$$
\left\{\begin{array}{l}
-\Delta W=A(x) W+F(x, U)+T \phi_{1}+H_{1}-A(x) U, \quad \Omega, \\
W=0, \quad \partial \Omega .
\end{array}\right.
$$

By (3.1), using the Maximum Principle for systems, we have that $\mathcal{T}$ is monotone. Let us define the sequences

$$
\left\{\begin{array}{rrr}
U_{0}=\underline{U}, & U_{n}=\mathcal{T} U_{n-1} \\
& \text { and } & \\
V_{0}=\bar{U}, & V_{n} & =\mathcal{T} V_{n-1}
\end{array}\right.
$$

By the previous arguments, we prove that $U_{1} \geq U_{0}$ and $V_{1} \leq V_{0}$. Iterating the above sequences we achieve that

$$
\underline{U}=U_{0} \leq U_{1} \leq U_{2} \leq \cdots \leq V_{2} \leq V_{1} \leq V_{0}=\bar{U} .
$$

So, there are pointwise convergent subsequences (denoted again by $U_{n}$ and $\left.V_{n}\right)$ such that

$$
U_{n} \rightarrow U \text { and } \quad V_{n} \rightarrow V \text { pointwise. }
$$

By a standard bootstrap argument we may assume the above convergences in $\left(C^{2, \alpha}\right)^{2}$. Hence, going to the limit in $(3.2)$ we conclude the first part of the proof. The second one is also proved by iteration.

Corollary 3.1. If (1.1), (1.6), (1.7), (1.8) and (3.1), hold and (S) has a solution for some $H \in\left(C^{\alpha}\right)^{2}$, then system $(S)$ has a minimal solution $U_{\text {min }}$. That is, if $U$ is another solution of $(S)$, then we have necessarily $U_{\min } \leq U$ in $\bar{\Omega}$.

Corollary 3.2. With the same assumptions of Corollary 3.1, if system $(S)$ has a solution for some $H_{1} \in\left(C^{\alpha}\right)^{2}$, then it has a solution for all $H_{2} \in\left(C^{\alpha}\right)^{2}$ with $H_{2} \leq H_{1}$.

Example 3.1. (An Application of Theorem 3.1) Consider the following problem

$$
\left\{\begin{aligned}
-\Delta u & =a(x) u+b(x) v-u^{3}, \quad \Omega, \\
-\Delta v & =c(x) u+d(x) v-v^{3}, \quad \Omega, \\
u & =v=0, \quad \partial \Omega .
\end{aligned}\right.
$$

Denote $A(x)=\left(\begin{array}{ll}a(x) & b(x) \\ c(x) & d(x)\end{array}\right)$ where $a, b, c, d \in C^{\alpha}(\bar{\Omega})$.

(i) If

$$
(A(x) Z, Z) \leq \underline{\mu}|Z|^{2} \text {, for some } 0<\underline{\mu}<\lambda_{1} \text {, all } x \in \Omega \text { and } Z \in \mathbb{R}^{2},
$$

then system (3.3) has unique solution $U=0$. 
(ii) If the matrix $A$ is cooperative and

$$
a(x), d(x)>\bar{\mu}>\lambda_{1},
$$

then system (3.3) has at least two non-trivial vector-solutions.

Proof. (i) Multiplying the first and the second equation in (3.3) by $u$ and $v$, respectively, integrating the expression by parts and using (3.4) and Poincaré inequality we have that

$$
\left(\lambda_{1}-\underline{\mu}\right) \int|U|^{2}+\int\left(u^{4}+v^{4}\right) \leq 0,
$$

and therefore that $U=0$.

(ii) If $\Phi=\left(\begin{array}{c}\phi_{1} \\ \phi_{1}\end{array}\right)$, using (3.5) one easily finds a subsolution $\sigma_{o} \Phi$ and a supersolution $\eta_{o} \Phi$ of system (3.3) for small $\sigma_{o} \in \mathbb{R}^{+}$and large $\eta_{o} \in \mathbb{R}^{+}$.

So $\sigma_{o} \Phi<\eta_{o} \Phi$. Define $N=\max _{x \in \bar{\Omega}}\left(\eta_{o} \Phi\right)$ and then choose $M>N$, such that $\left(A_{M}(x) Z, Z\right) \leq \mu_{*}|S|^{2}$ for some $0<\mu_{*}<\lambda_{1}$ and all $Z \in \mathbb{R}^{2}, x \in \Omega$, where

$$
A_{M}(x)=\left(\begin{array}{cc}
a(x)-3 M^{2} & b(x) \\
c(x) & d(x)-3 M^{2}
\end{array}\right) .
$$

In this way, if $U$ satisfies $0<\sigma_{o} \Phi \leq U \leq \eta_{o} \Phi<\left(\begin{array}{l}M \\ M\end{array}\right)$, defining $f(x, u, v)=$ $a(x) u+b(x) v-u^{3}$ and $g(x, u, v)=c(x) u+d(x) v-v^{3}$, we have that $F\left(x, U_{2}\right)-F\left(x, U_{1}\right) \geq A_{M}(x)\left(U_{2}-U_{1}\right) \quad$ for $\quad U_{2} \geq U_{1} ; U_{1}, U_{2} \in\left[\sigma_{o} \Phi, \eta_{o} \Phi\right]$.

Now by Remark 2.1 and the previous theorem there exists a solution $U \in$ $\left(C^{2, \alpha}\right)^{2}$ of system (3.3). The vector- function $-U$ is the other solution.

Remark 3.1. Observe that

$$
(A(x) Z, Z) \geq \bar{\mu}|Z|^{2}, \quad \text { for some } \bar{\mu}>\lambda_{1}, \quad \forall x \in \Omega, Z \in \mathbb{R}^{2},
$$

is a symmetric condition to (3.4) and implies (3.5).

\section{The Curve}

Let us now construct the curve. For this purpose, let us fix $H_{1}=\left(\begin{array}{l}h_{1} \\ l_{1}\end{array}\right) \in N_{\perp}^{2}$ and define

and

$$
W_{t}:=\left\{(r, s) \in \mathbb{R}^{2}, s+t=r\right\}
$$

$R(t)=\left\{r \in \mathbb{R} ;(S)_{T}\right.$ has a solution with $T \in W_{t}$ for some $\left.s \in \mathbb{R}\right\}$.

By Lemma 1.1 and Theorem 3.1, the curve below is well-defined:

$$
\Gamma(t)=(\sup R(t), \sup R(t)-t)=(r(t), s(t)), t \in \mathbb{R} .
$$

It is readily seen the following properties of $\Gamma$ :

$\left.\Gamma_{1}\right) \Gamma$ increases in the $r$ direction and decreases in the $s$ direction (ie, $t^{\prime}<t^{\prime \prime}$ implies $r\left(t^{\prime}\right) \leq r\left(t^{\prime \prime}\right)$ and $\left.s\left(t^{\prime}\right) \geq s\left(t^{\prime \prime}\right)\right)$.

$\left.\Gamma_{2}\right) r\left(t^{\prime \prime}\right)-r\left(t^{\prime}\right) \leq t^{\prime \prime}-t^{\prime}$ for $t^{\prime} \leq t^{\prime \prime}$.

$\left.\Gamma_{3}\right) \Gamma$ is a Lipschitzian curve.

$\left.\Gamma_{4}\right) \lim _{t \rightarrow-\infty} r(t)=-\infty$ and $\lim _{s \rightarrow+\infty} s(t)=-\infty$. 
for $\bar{b}, \underline{b} \neq 0$.

From all our results so far and from the construction of $\Gamma$ we are now able to state the final Theorem of the first part.

Theorem 4.1. Suppose that (1.1), (1.2), (1.5), (1.6), (1.7), (1.8) and (3.1) hold. Then for each fixed $H_{1} \in\left(C^{\alpha}\right)^{2}$ there exists a Lipschitzian curve $\Gamma \in$ $\mathbb{R}^{2}$ splitting the plane into two unbounded domains $E$ and $N\left(\mathbb{R}^{2}=E \cup \Gamma \cup N\right.$ where $N$ is the part of the plane above $\Gamma$ ) such that

(i) $(S)_{T}$ has no solution if $T \in N$.

(ii) $(S)_{T}$ has at least one solution if $T \in \Gamma$.

Remark 4.1. Changing the previous order in $\mathbb{R}^{2}$ by the new order $U \geq 0$ if and only if $u \geq 0$ and $v \leq 0$, mutatis mutandis previous definitions and hypotheses, a similar theorem to Theorem 4.1still holds when the matrices $A$ and $\underline{A}$ are not cooperative, with non-positive off diagonal terms. In this case a similar curve to $\Gamma$ is found with similar properties.

To finish this section, we give

Example 4.1. (The Ambrosetti-Prodi problem for the biharmonic equation) Let us consider the biharmonic equation

$$
\left\{\begin{aligned}
(\Delta+a)(\Delta+b) v & =f(x, v)+t \phi_{1}+h(x), \quad \Omega, \\
\Delta v=v & =0, \quad \partial \Omega
\end{aligned}\right.
$$

with $a, b, t \in \mathbb{R}$. This equation is equivalent to the elliptic system

$$
\left\{\begin{aligned}
-\Delta u & =a u+f(x, v)+t \phi_{1}+h, \quad \Omega, \\
-\Delta v & =u+b v, \quad \Omega \\
u & =v=0, \quad \partial \Omega .
\end{aligned}\right.
$$

If the following hypotheses hold:

$$
-\infty \leq \limsup _{s \rightarrow-\infty} \frac{f(x, s)}{s}<\left(\lambda_{1}-a\right)\left(\lambda_{1}-b\right)<\liminf _{s \rightarrow+\infty} \frac{f(x, s)}{s} \leq+\infty
$$

uniformly for all $x \in \Omega$ and

$$
f\left(x, s_{2}\right)-f\left(x, s_{1}\right) \geq k\left(s_{2}-s_{1}\right), s_{2}, s_{1} \in \mathbb{R}, s_{2} \geq s_{1},
$$

with $k \in \mathbb{R}^{+}$such that (1.6), (1.7) and (1.8) hold for the matrix $A=$ $\left(\begin{array}{cc}a & k \\ 1 & b\end{array}\right)$, then we have that there exists $t_{o} \in \mathbb{R}$ such that

(i) Problem (4.1) has no solution, if $t>t_{o}$

(ii) Problem (4.1) has at least one solution if $t<t_{o}$.

\section{SECOND PART}

In this second part we shall improve Theorem 4.1 by finding another solution if $T \in E$ and a solution if $T \in \Gamma$. 


\section{A PRIORI Bounds}

The a priori estimates we are to obtain are needed to the use of the Leray-Schauder Degree. In the following section we apply degree theory in order to find the other solution. In this section we use the ideas of BrèzisTurner [2], where they made estimates in the scalar case using the HardySobolev inequality (see [2] or [6] ). In our work the limitations of the growth conditions on the non-linearities $f$ and $g$ ( see (5.16) and (5.17) below ) are due to the restriction of the exponents of the Hardy-Sobolev inequality. Let us denote by $U=(u, v)$ an eventual solution of the system

$$
\left\{\begin{array}{l}
-\Delta U=F(x, U)+H(x), \quad \Omega, \\
U=0, \quad \partial \Omega .
\end{array}\right.
$$

The same letter $c$ will denote a generic positive constant.

Lemma 5.1. ( $L_{\text {loc }}^{1}$ estimates) If assumptions (1.1), (1.2), (1.4) and (1.5) hold, then there exists a constant $c>0$, depending on $H, \underline{A}$ and $\bar{A}$, such that

$$
\int\left|u \phi_{1}\right|, \int\left|v \phi_{1}\right| \leq c
$$

Proof. As in Lemma 1.1, using assumptions (1.1) and (1.2) we obtain

$$
\left(\underline{A}-\lambda_{1} I\right)\left(\begin{array}{c}
\int u \phi_{1} \\
\int v \phi_{1}
\end{array}\right) \leq\left(\begin{array}{c}
c \\
c
\end{array}\right),
$$

and

$$
\left(\bar{A}-\lambda_{1} I\right)\left(\begin{array}{c}
\int u \phi_{1} \\
\int v \phi_{1}
\end{array}\right) \leq\left(\begin{array}{c}
c \\
c
\end{array}\right),
$$

where $I$ is the $2 \times 2$ identity matrix.

Applying $\left(\underline{A}-\lambda_{1} I\right)^{-1}$ and $\left(\bar{A}-\lambda_{1} I\right)^{-1}$ to (5.3) and (5.4), respectively, and using (1.4) and (1.5) we get (5.2) for some $c \in \mathbb{R}^{+}$.

From Corollary 3.1 it is readily seen that the following lemma holds.

Lemma 5.2. (A priori estimates for the negative part) Suppose that assumptions (1.1), (1.2), (1.5), (1.6), (1.7), (1.8) and (3.1) hold. Then there exists $c>0$ such that

$$
\left\|u^{-}\right\|_{L^{\infty}(\Omega)},\left\|v^{-}\right\|_{L^{\infty}(\Omega)} \leq c,
$$

where $u^{-}:=\max (0,-u)$.

To proceed further, we need the following result.

Lemma 5.3. Suppose that (1.1), (1.2), (1.5), (1.6), (1.7), (1.8) and (3.1) hold. Then there exists $c>0$ such that

$$
\int|f(x, u, v)| \phi_{1}, \int|g(x, u, v)| \phi_{1} \leq c .
$$


Proof. From Lemmas (5.1) and (5.2) we have

$$
\int u^{+} \phi_{1}, \int u^{-} \phi_{1}, \int v^{+} \phi_{1}, \int v^{-} \phi_{1} \leq c .
$$

Let $\Omega^{-}=\{x \in \Omega ; f(x, u(x), v(x)) \leq 0\}$ and $\Omega^{+}=\Omega / \Omega^{-}$. Assumption (1.1) and the relations (5.7) imply that

$$
-\int_{\Omega^{-}} f(x, u, v) \phi_{1} \leq-\underline{a} \int_{\Omega^{-}} u \phi_{1}-\underline{b} \int_{\Omega^{-}} v \phi_{1}+c \leq c .
$$

Observe that

$$
\lambda_{1} \int u \phi_{1}=\int\left(-\Delta u \phi_{1}\right)=\int f \phi_{1}+\int h \phi_{1}
$$

and then, by (5.2):

$$
\left|\int f \phi_{1}\right| \leq c \quad \text { where } \quad(c=c(h)) .
$$

Therefore, from (5.8) and (5.9),

$$
\int_{\Omega}|f| \phi_{1}=\int_{\Omega^{+}} f \phi_{1}-\int_{\Omega^{-}} f \phi_{1}=\int_{\Omega} f \phi_{1}-2 \int_{\Omega^{-}} f \phi_{1} \leq c .
$$

A similar estimate can be obtained with $g$ replacing $f$.

Remark 5.1. The constant $c$ appearing in (5.9) - and hence in (5.6) depends upon $H$ but it is independent of $U$. It is relevant to remark that this constant may be taken uniformly with respect to $H$ varying in a bounded subset of $\left(C^{\alpha}\right)^{2}$. Later we shall make use of this important fact.

For estimating purposes we need the following growth conditions on the non-linearities, for all $x \in \Omega ; \eta, \xi \in \mathbb{R}$ :

$$
\begin{gathered}
f(x, \eta, \xi)=f_{1}(x, \eta)+f_{2}(x, \xi) ; \quad f_{1}, f_{2} \in C^{\alpha}(\bar{\Omega} \times \mathbb{R}, \mathbb{R}), \\
\lim _{\eta \rightarrow+\infty} \frac{f_{1}(x, \eta)}{\eta^{\frac{N+1}{N-1}}}=0, \quad \text { uniformly for } x \in \bar{\Omega}, \\
\left|f_{2}(x, \xi)\right| \leq c\left(|\xi|^{p}+1\right), \quad p \text { as restricted below, } \\
g(x, \eta, \xi)=g_{1}(x, \xi)+g_{2}(x, \eta) ; \quad g_{1}, g_{2} \in C^{\alpha}(\bar{\Omega} \times \mathbb{R}, \mathbb{R}), \\
\lim _{\xi \rightarrow+\infty} \frac{g_{1}(x, \xi)}{\xi^{\frac{N+1}{N-1}}}=0, \quad \text { uniformly for } x \in \bar{\Omega}, \\
\left|g_{2}(x, \eta)\right| \leq c\left(|\eta|^{q}+1\right), \quad q \text { as restricted below, } \\
0 \leq p q<\left(\frac{N+2}{N}\right)^{2} \text { and } \\
1 \leq p, q \leq \frac{N}{N-2} \text { if } N>3, \text { and } 1 \leq p, q<\infty \text { if } N=2
\end{gathered}
$$


Remark 5.2. In our estimates, $p$ and $q$ are restricted to be below the socalled critical hyperbola: $\frac{1}{p+1}+\frac{1}{q+1}>\frac{N-2}{N}$. However, our estimates include some cases that are not covered in the papers [3] and [9], where $a$ priori estimates are also obtained. It is also important to point out that, contrary the scalar case, in our work the exponents $p$ or $q$ may grow beyond the Brèzis-Turner exponent: $\frac{N+1}{N-1}$.

Theorem 5.1. ( $H_{0}^{1}(\Omega)$-a priori estimate for the positive part) If the assumptions (1.1), (1.2), (1.5), (1.6), (1.7), (1.8), (3.1), (5.10),.., (5.16) and (5.17) hold, then there exists a constant $c>0$ such that

$$
\left\|u^{+}\right\|_{H_{0}^{1}(\Omega)},\left\|v^{+}\right\|_{H_{0}^{1}(\Omega)} \leq c .
$$

Proof. There is no loss of generality in assuming that $f_{1}(x, \eta) \geq 0$ for $\eta \geq 0$ and $f_{2}(x, \xi) \geq 0$ for $\xi \geq 0$. Indeed, using (5.10) and (1.1) we have $f_{1}(x, \eta) \geq$ $\underline{a} \eta-c^{\prime}$ for $\eta \geq 0$. Let $m_{1}>c^{\prime}$. Let us consider $\widetilde{f}_{1}=f_{1}+m_{1} \geq 0$ and $\widetilde{\ell}=\ell-m_{1}$ in (5.10). Then $\tilde{f}_{1}$ satisfies all the hypotheses required for $f_{1}$. The same is done for $f_{2}$. Hence hereafter we shall assume that $f_{1}, f_{2} \geq 0$ for $\eta \geq 0$ and $\xi \geq 0$, respectively. We may write

$$
\begin{aligned}
f(x, u, v)= & f\left(x, u^{+}, v^{+}\right)+f\left(x, u^{+},-v^{-}\right)+f\left(x,-u^{-}, v^{+}\right) \\
& +f\left(x,-u^{-},-v^{-}\right)-f\left(x, 0,-v^{-}\right)-f\left(x,-u^{-}, 0\right) \\
& -f\left(x, u^{+}, 0\right)-f\left(x, 0, v^{+}\right)+f(x, 0,0) .
\end{aligned}
$$

The above facts with (5.5), (5.6) and (5.10) lead to

$$
\int\left|f_{1}\left(x, u^{+}\right)\right| \phi_{1}, \int\left|f_{2}\left(x, v^{+}\right)\right| \phi_{1} \leq c
$$

for some $c>0$. Multiplying the first equation of (5.1) by $u^{+}$, then integrating by parts the resulting expression and using the a priori estimates for the negative parts (5.5), we obtain

$$
\begin{aligned}
\left\|\nabla u^{+}\right\|_{L^{2}}^{2} & \leq c\left(\int\left|f_{1}\left(x, u^{+}\right)\right| u^{+}+\int\left|f_{2}\left(x, v^{+}\right)\right| u^{+}+\left\|\nabla u^{+}\right\|_{L^{2}}\right) \\
& =c\left(I_{1}+I_{2}+\left\|\nabla u^{+}\right\|_{L^{2}}\right) .
\end{aligned}
$$

Now let us estimate $I_{1}$ and $I_{2}$, using the Hardy-Sobolev Inequality (see [2]):

By virtue of (5.11) and (5.20), for an arbitrary $\varepsilon>0$ we have

$$
I_{1} \leq c(\varepsilon)\left\|\nabla u^{+}\right\|_{L^{2}}^{2}+C_{\varepsilon}\left\|\nabla u^{+}\right\|_{L^{2}},
$$

where $c(\varepsilon)=0(1)$ as $\varepsilon \rightarrow 0$ and $C_{\varepsilon}>0$ (see Lemma 2.4 in [2]). The proof of this inequality follows the same ideas that we are going to use in the following to estimate $I_{2}$. Hence we suppress it here.

In order to estimate $I_{2}$, let us we consider $0<\theta<1$ to be chosen later. Hölder inequality implies that

$$
I_{2} \leq\left(\int\left|f_{2}\left(x, v^{+}\right)\right| \phi_{1}\right)^{\theta}\left(\int \frac{\left|f_{2}\left(x, v^{+}\right)\right|\left(u^{+}\right)^{1 /(1-\theta)}}{\phi_{1}^{\theta /(1-\theta)}}\right)^{1-\theta} .
$$


It follows from (5.12) and (5.20) that

$$
\begin{aligned}
I_{2} & \leq c\left\{\left(\int \frac{\left(v^{+}\right)^{p}\left(u^{+}\right)^{1 /(1-\theta)}}{\phi_{1}^{\theta /(1-\theta)}}\right) 1-\theta+\left(\int \frac{\left(u^{+}\right)^{1 /(1-\theta)}}{\phi_{1}^{\theta /(1-\theta)}}\right){ }^{1-\theta}\right\} \\
& =c\left(I_{3}+I_{4}\right) .
\end{aligned}
$$

From the Hardy-Sobolev Inequality, it follows that

$$
I_{4} \leq c\left\|\nabla u^{+}\right\|_{L^{2}} \quad \text { if } \quad \frac{1}{1-\theta} \leq R \quad \text { and } \quad \frac{1}{R}=\frac{1}{2}-\frac{(1-\theta)}{N} .
$$

On the other hand,

$$
I_{3} \leq C_{\varepsilon}^{\prime}\left\|v^{+}\right\|_{L^{2 p}}^{2 p(1-\theta)}+c^{\prime}(\varepsilon)\left\|\frac{u}{\phi_{1}^{\theta}}\right\|_{L^{2 /(1-\theta)}}^{2} .
$$

In view of (5.17) and the Sobolev Embbeding Theorem, we have that

$$
\left\|v^{+}\right\|_{L^{2 p}}^{2 p(1-\theta)} \leq c\left\|\nabla v^{+}\right\|_{L^{2}}^{2 p(1-\theta)} .
$$

Again, by the Hardy-Sobolev Inequality and the Sobolev Embedding Theorem, if

$$
\frac{2}{1-\theta} \leq Q \quad \text { and } \quad \frac{1}{Q}=\frac{1}{2}-\frac{(1-\theta)}{N}
$$

then

$$
\left\|\frac{u^{+}}{\phi_{1}^{\theta}}\right\|_{L^{2 /(1-\theta)}}^{2} \leq c\left\|\nabla u^{+}\right\|_{L^{2}}^{2} .
$$

Thereby, from $(5.21), \ldots,(5.27)$, we have

$$
\left\|\nabla u^{+}\right\|_{L^{2}} \leq c\left(\left\|\nabla v^{+}\right\|_{L^{2}}^{p(1-\theta)}+\left\|\nabla u^{+}\right\|_{L^{2}}^{1 / 2}\right) .
$$

A similar reasoning yields

$$
\left\|\nabla v^{+}\right\|_{L^{2}} \leq c\left(\left\|\nabla u^{+}\right\|_{L^{2}}^{q(1-\theta)}+\left\|\nabla v^{+}\right\|_{L^{2}}^{1 / 2}\right) .
$$

Choosing $\theta=\frac{2}{N+2}$, (the best possible for our purposes), using (5.16), (5.28), (5.29) and the assertion below, the estimates (5.18) are attained.

Assertion. If $a, b \geq 0$ are such that $a \leq c\left(a^{\gamma_{1}}+b^{\gamma_{2}}\right), b \leq c\left(a^{\gamma_{3}}+b^{\gamma_{4}}\right)$ for some $c>0$, with $0<\gamma_{1}, \gamma_{3}<1$ and $0<\gamma_{2} \gamma_{4}<1$, then there exists $K \geq 0$ such that $a, b \leq K$.

As the growth condition on the non-linearities $f$ and $g$ are subcritical, a bootstrap argument enable us to obtain estimates in spaces of more regular functions. Indeed, from Lemma 5.2 and Theorem 5.1, we have

Corollary 5.1. $\left(C^{1, \alpha}(\Omega)\right.$-a priori estimates) With the assumptions of Theorem 5.1, there exists a constant $c>0$ such that

$$
\|u\|_{C^{1, \alpha}(\Omega)},\|v\|_{C^{1, \alpha}(\Omega)} \leq c .
$$




\section{Computation of Some Topological Degrees}

As previously said, in this section, employing Leray-Schauder degree Theory, we shall improve Theorem 4.1 by finding another solution of system $(S)$ if $T \in E$ and one solution if $T$ is on the curve $\Gamma$. A priori estimates are required for the use of topological degree. So, throughout this section we shall assume all the hypotheses of Theorem 5.1, where a priori bounds were found, with the additional hypothesis that $A$ in (3.1) is cooperative.

First, for each $T=\left(\begin{array}{l}r \\ s\end{array}\right) \in \mathbb{R}^{2}$ let us define the operator

$$
\begin{aligned}
K_{T}:\left(C^{1, \alpha}\right)^{2} & \longrightarrow\left(C^{2, \alpha}\right)^{2} \\
U & \longmapsto V
\end{aligned}
$$

where $V$ is the unique solution (see Remark 3.1) of the system

$$
\left\{\begin{array}{l}
-\Delta V=A(x) V+F(x, U)-A(x) U+T \phi_{1}+H_{1}, \quad \Omega, \\
V=0, \quad \Omega .
\end{array}\right.
$$

( $A$ is the same matrix that appears in Theorem 3.1).

From Remark 0.1 and Remark 2.2, it follows that $K_{T}$ is a compact operator if looked from $\left(C^{1, \alpha}\right)^{2}$ into itself.

With this setting, $U$ is a solution of $(S)$ if, and only if $\left(I-K_{T}\right) U=0$. Therefore Leray-Schauder degree is applicable to find a solution of the last equation.

Lemma 6.1. Let $T_{o} \in \mathbb{R}^{2}$ and $H_{1} \in N_{\perp}^{2}$ be fixed. Then there exists a constant $R>0$ such that

$$
\begin{gathered}
D\left(I-K_{T_{o}}, B_{R}(0), 0\right)=0, \quad \text { where } \\
B_{R}(0)=\left\{U \in\left(C^{1, \alpha}\right)^{2} ;\|U\|_{\left(C^{1, \alpha}\right)^{2}}<R\right\}
\end{gathered}
$$

Proof. $T_{0} \in W_{t_{0}}$ for some $t_{0} \in \mathbb{R}$. Fix some $T_{1} \in W_{t_{0}} \cap N$ and let $\widetilde{W}_{t_{0}}$ be the closed segment joining the points $T_{0}$ and $T_{1}$. From Corollary 5.1 and Remark 5.1, there exists $R>0$ such that $\|U\|_{\left(C^{1, \alpha}\right)^{2}}<R$ for any eventual solution of $(S)_{T}$ for all $T \in \widetilde{W}_{t_{0}}$. Since $K_{T}$ is an admissible homotopy joining $K_{T_{0}}$ and $K_{T_{1}}$ and $(S)_{T_{1}}$ has no solution, we obtain (6.3).

Lemma 6.2. Given $T_{0} \in E$ and $H_{1} \in N_{\perp}^{2}$, there exists an open, bounded and convex subset $\mathcal{O} \subseteq\left(C^{1, \alpha}\right)^{2}$ such that

$$
D\left(I-K_{T_{0}}, \mathcal{O}, 0\right)=1 .
$$

Proof. $T_{0} \in W_{t_{0}}$ some $t_{0} \in \mathbb{R}$. Let $\dot{W}_{t_{0}}$ be the open segment on $W_{t_{0}}$ joining $T_{0}$ to $\Gamma\left(t_{0}\right)$. Fixed $T_{1} \in \dot{W}_{t_{0}}$, it follows from Theorem 4.1 that system $(S)_{T_{1}}$ has a solution $\bar{U}=\left(\begin{array}{l}\bar{u} \\ v\end{array}\right)$ which is a supersolution for $(S)_{T_{0}}$. By Lemma 2.1 there exists a subsolution $\underline{U}=\left(\frac{u}{v}\right)$ for $(S)_{T_{0}}$, such that $\underline{U}<\bar{U}$ in $\Omega$ and 
$\frac{\partial \bar{U}}{\partial \nu}<\frac{\partial \underline{U}}{\partial \nu}$ on $\partial \Omega$. The set $\mathcal{O}$ is defined by

$$
\mathcal{O}=\left\{U \in\left(C^{1, \alpha}(\bar{\Omega})\right)^{2} ; \underline{U}<\bar{U} \text { in } \bar{\Omega}, \frac{\partial \bar{U}}{\partial \nu}<\frac{\partial \underline{U}}{\partial \nu} \text { on } \partial \Omega\right.
$$

and $\left.\|U\|_{\left(C^{1, \alpha}\right)^{2}}<\mathrm{r}\right\}$ for some $\mathrm{r}>0$ to be chosen later.

Let us define

$$
\tilde{f}(x, \eta, \xi)=\left\{\begin{array}{cccc}
f(x, \underline{u}(x), \underline{v}(x))-a(x) \underline{u}(x)-b(x) \underline{v}(x), & \text { if } & & \eta \leq \underline{u}(x) \\
f(x, \eta, \xi)-a(x) \eta-b(x) \xi, & \text { or } & & \xi \leq \underline{v}(x) \\
& \text { if } & \underline{u}(x) \leq \eta \leq \bar{u}(x) \\
f(x, \bar{u}(x), \bar{v}(x))-a(x) \bar{u}(x)-b(x) \bar{v}(x), & \text { if } & \text { ind } & \underline{v}(x) \leq \xi \leq \bar{v}(x) \\
& \text { or } & & \xi \geq \bar{u}(x)
\end{array}\right.
$$

In a similar way we define a function $\widetilde{g}$. The functions $\widetilde{f}$ and $\widetilde{g}$ are bounded and non-decreasing. With these functions we can introduce, as in the beginning of this section, the operator $\widetilde{K}_{T_{0}}:\left(C^{1, \alpha}\right)^{2} \mapsto\left(C^{2, \alpha}\right)^{2}$ such that $\widetilde{K}_{T_{0}} U=V$ where $V$ is the unique solution of the system

$$
\left\{\begin{array}{l}
-\Delta V=A(x) V+\widetilde{F}(x, U)+T_{0} \phi_{1}+H_{1}, \quad \Omega, \\
V=0, \quad \partial \Omega .
\end{array}\right.
$$

As before, $\widetilde{K}_{T_{0}}$ is a compact operator of $\left(C^{1, \alpha}\right)^{2}$ into itself.

Since $\tilde{f}$ and are bounded, it follows from $L^{p}$ estimates for systems that all the solutions of (6.6) are uniformly bounded in $\left(C^{1, \alpha}\right)^{2}$. So, if we choose $\mathrm{r}>\sup \left\{\left\|K_{T_{0}} U\right\|_{\left(C^{1, \alpha}\right)^{2}} ; U \in\left(C^{1, \alpha}\right)^{2}\right\}$, use (3.1), use the fact that $\tilde{f}$ and $\tilde{g}$ are non-decreasing, and use the Maximum Principle for systems, it follows that $\widetilde{K}_{T_{0}}(\overline{\mathcal{O}}) \subseteq \mathcal{O}$.

Now take $\psi \in \mathcal{O}$ and consider the admissible compact homotopy $H_{\theta}(U)=$ $\theta \widetilde{K}_{T_{0}}(U)+(1-\theta) \psi, 0 \leq \theta \leq 1$. As $\psi$ is a constant mapping, $D\left(I-H_{0}, \mathcal{O}, 0\right)=$ 1 and hence $D\left(I-\widetilde{K}_{T_{0}}, \mathcal{O}, 0\right)=1$. The proof is finished since $\left.\widetilde{K}_{T_{0}}\right|_{\mathcal{O}} \equiv$ $K_{T_{0}}$.

Theorem 6.1. Consider the assumptions of Theorem 5.1. Then the conclusions of Theorem 4.1 can be strengthened as follows:

(i) $(S)_{T}$ has no solution if $T \in N$

(ii) $(S)_{T}$ has at least one solution if $T \in \Gamma$

(iii) $(S)_{T}$ has at least two solutions if $T \in E$.

Proof. Case (i) is already contained in Theorem 4.1. Case (ii) is consequence of Lemmas 6.1 and 6.2. Indeed take $R>0$ in Lemma 6.1 such that $R>\mathrm{r}$. Since $D\left(I-K_{T_{0}}, \mathcal{O}, 0\right)=1$ we have a solution $U$ in $\mathcal{O}$. By Lemma 6.1 and excision property of the degree we have that $D\left(I-K_{T}, B_{R}(0) / \mathcal{O}, 0\right)=-1$ and then there exists another solution $W \neq U$ of $(S)_{T}$ in $B_{R}(0) / \mathcal{O}$. In Case (iii), take a sequence $\left(T_{n}\right) \subseteq E$ such that $T_{n} \rightarrow T$. By the uniform a priori bound (Corollary 5.1 and Remark 5.1 ) we have that $\left\|U_{n}\right\|_{\left(C^{1, \alpha}\right)^{2}} \leq c$ for some $c>0$. By the compact embedding Theorem (Remark 0.1) we have (passing eventually to a subsequence) $U_{n} \rightarrow U_{0}$ in $\left(C^{\alpha}\right)^{2}$ for some $U_{0} \in\left(C^{\alpha}\right)^{2}$. 
Using Schauder estimates for systems we have the above convergence in $\left(C^{2, \alpha}\right)^{2}$ and then going to the limit in $(S)_{T_{n}}$ we finish the proof of (iii).

Example 6.1. (Where Theorem 6.1 holds) Let us consider system $(S)$ with $f=f(x, v)$ and $g=g(x, u)$.

Suppose that $f$ and $g$ are non-decreasing functions ( hence (3.1) holds ) and that (5.12), (5.15) and (5.16) are satisfied. If in addition the following assumptions hold

$$
\begin{aligned}
& -\infty \leq \limsup _{v \rightarrow-\infty} \frac{f(x, v)}{v}<\Lambda_{f}<\liminf _{v \rightarrow+\infty} \frac{f(x, v)}{v} \leq+\infty \\
& -\infty \leq \limsup _{u \rightarrow-\infty} \frac{g(x, u)}{u}<\Lambda_{g}<\liminf _{u \rightarrow+\infty} \frac{g(x, u)}{u} \leq+\infty
\end{aligned}
$$

uniformly for $x \in \Omega$, where

$$
\Lambda_{f}, \Lambda_{g}>0 \text { and } \Lambda_{f} \Lambda_{g}=\lambda_{1}^{2},
$$

then (1.1), (1.2), (1.6), (1.7), (1.8), (1.15), (1.16) and (1.17) are valid. Therefore Theorem 6.1 holds for system $(S)$ with the above non-linearities.

Note that conditions (6.7) and (6.8) generalizes for systems the condition

$$
-\infty \leq \limsup _{u \rightarrow-\infty} \frac{f(x, u)}{u}<\lambda_{1}<\liminf _{u \rightarrow+\infty} \frac{f(x, u)}{u} \leq+\infty
$$

uniformly for $x \in \Omega$, asked in the scalar case (see [4] on [8]).

\section{REFERENCES}

[1] A. Ambrosetti and G. Prodi, On the inversion of some Differentiable mappings with singularities between Banach spaces, Ann. Math. Pura Appl. 93 (1972), 231-247.

[2] H. Brézis and R. E. L. Turner, On a class of superlinear elliptic problems, Comm. Partial Differential Equations, 2 (1977), 601-614.

[3] P. Clément, D. G. de Figueiredo and E. Mitidieri, A priori estimates for positive solutions of semilinear elliptic systems via Hardy-Sobolev inequalities, Technical Report, \#20, UNICAMP, 1992.

[4] D. G. de Figueiredo, Lectures on Boundary Value Problems of Ambrosetti-Prodi Type, $12^{\text {th }}$ Brazilian Seminar of Analysis, São Paulo, Brasil, 1980.

[5] J. Fleckinger, J. Hernandez and F. de Thélin, On maximum principles and existence of positive solutions for some cooperative elliptic systems, Preprint, May 1992.

[6] O. Kavian, Inegalité de Hardy-Sobolev et Application, Thése de Doctorat de $3^{\text {eme }}$ Cycle, Université de Paris VI, 1978.

[7] D. C. de Morais Filho, A variational approach to an Ambrosetti-Prodi type problem for a system of elliptic equations, Nonlinear Anal. 26 (1996), 1655-1668.

[8] M. Ramos, Teoremas de multiplicidade do tipo Ambrosetti-Prodi, Textos e Notas, CMAF, Lisboa-Portugal, 1988.

[9] M. A. S. Souto, A Priori estimates and existence of positive solutions of nonlinear cooperative elliptic systems, Differential Integral Equations, 8 (1995), 1245-1258.

[10] M. A. S. Souto and F. J. S. A. Correa, On maximum principles for cooperative elliptic systems via fixed point theory, Nonlinear Anal. 26 (1996), 997-1006.

Departamento de Matemática e Estatística, Universidade Federal da Paraíba, Caixa Postal 10044, 58.109-970 - Campina Grande - Pb - Brazil

E-mail address:daniel@dme.ufpb.br 


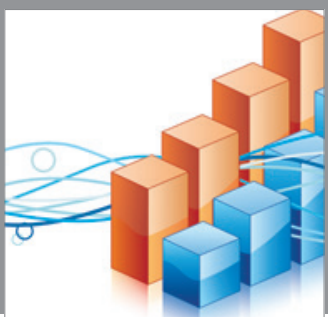

Advances in

Operations Research

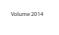

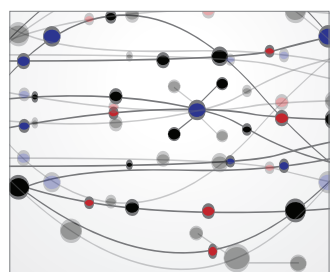

\section{The Scientific} World Journal
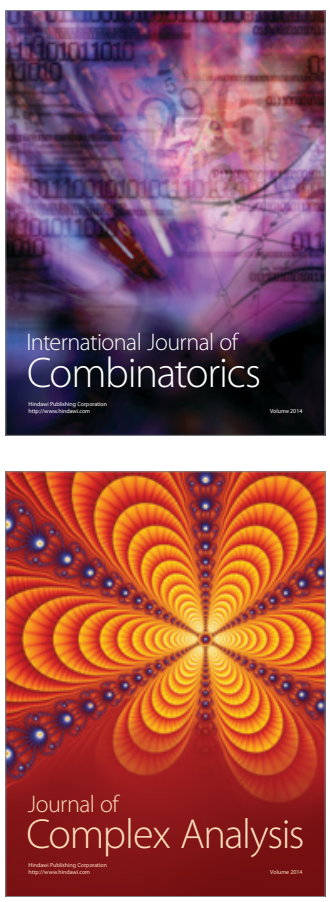

International Journal of

Mathematics and

Mathematical

Sciences
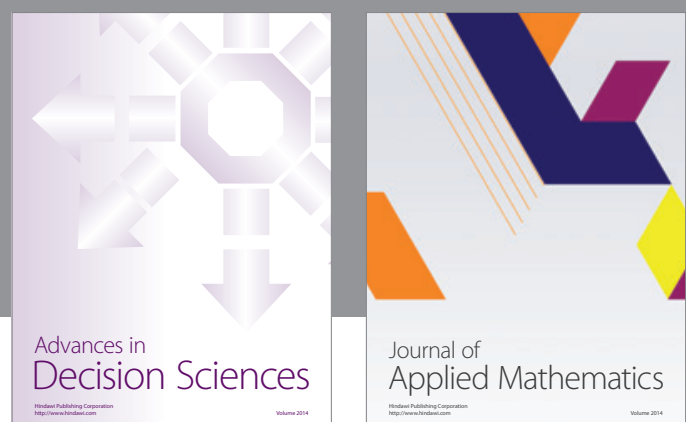

Journal of

Applied Mathematics
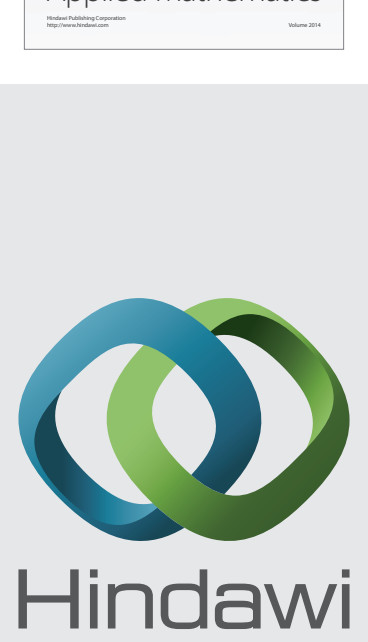

Submit your manuscripts at http://www.hindawi.com
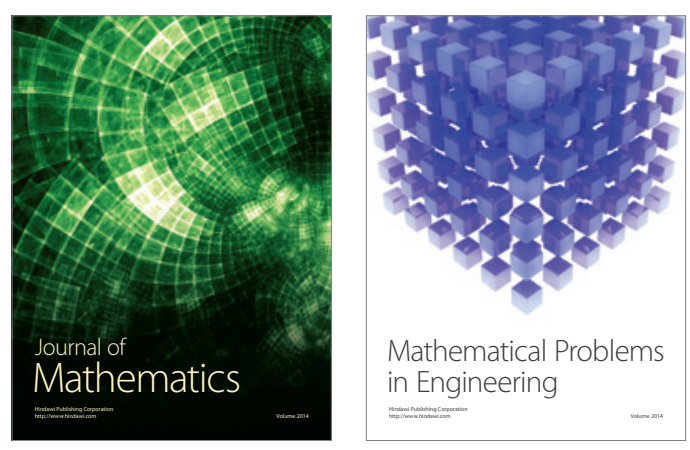

Mathematical Problems in Engineering
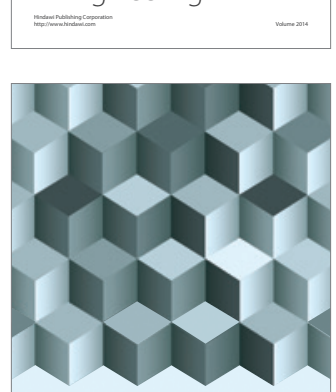

Journal of

Function Spaces
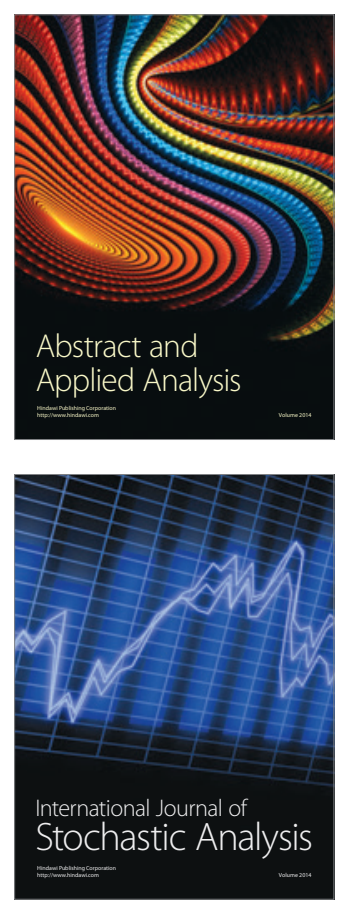

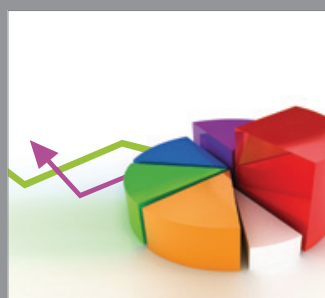

ournal of

Probability and Statistics

Promensencen
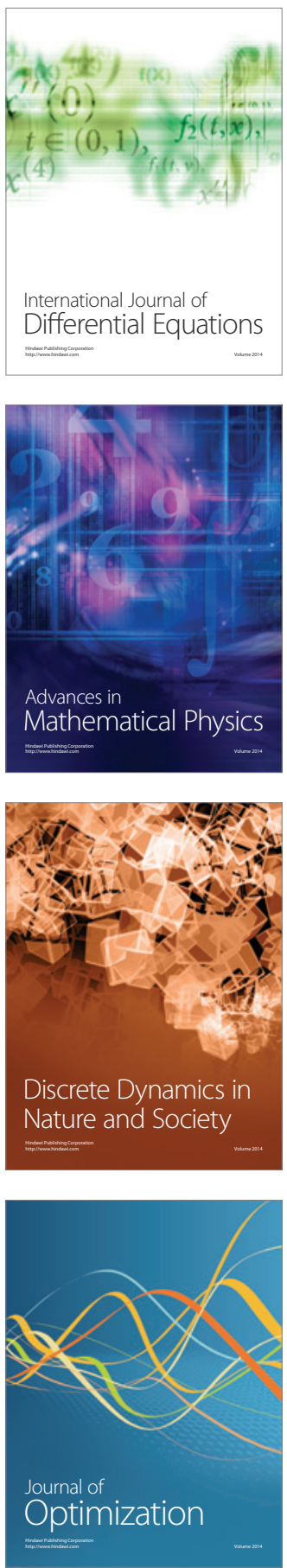